\title{
Maternal surfactant protein A influences the immunoprotective properties of milk in a murine model
}

\author{
Monisha P. Gidvani' ${ }^{1}$ Erin Theisen ${ }^{2}$, Robert Leduc ${ }^{3}$ and Caroline L.S. George ${ }^{2}$
}

BACKGROUND: Maternal surfactant protein A (SP-A), a collectin with innate immune system function, is critical to newborn mouse survival preventing bacterial peritonitis associated with a nonhygienic environmental exposure. We hypothesized that SP-A improves newborn survival by optimizing milk immunoprotection.

METHODS: Regional (lung) and systemic (milk and serum) immunologic responses to a novel antigen, 2,4-dintirophenyl keyhole limpet hemocyanin (DNP-KLH), and to a nonhygienic environment were evaluated in wild-type (WT) and SP-A null murine dams. Cross-fostering pups assessed the impact of milk on newborn survival.

RESULTS: Maternal SP-A optimized antigen-specific milk secretory $\lg A(s \lg A)$ production following the DNP-KLH exposure. Milk total and environment-specific slgA production was not dependent on maternal SP-A in the nonhygienic exposure. At baseline, SP-A null milk contained physiologically meaningful increases in two proinflammatory cytokines compared with WT milk. The lack of SP-A plus the nonhygienic environmental exposure synergistically increased the number of proinflammatory cytokines contained in milk. Finally, the SP-A null genotype decreased pup survival during a nonhygienic environmental exposure.

CONCLUSION: Maternal SP-A impacts milk slgA and cytokine content, and is associated with improved newborn health.

C ollectins are proteins with innate immune system functions, characterized by collagenous- and carbohydratebinding domains. Surfactant protein A (SP-A) is the most abundant collectin in the aqueous lining of the respiratory track (1). SP-A null mice have an exaggerated proinflammatory cytokine response following a bacterial infection and allergen stimulation, yet do not require pathogen-free conditions to survive into adulthood and to reproduce $(2,3)$. When purified SP-A is added to in vitro alveolar macrophages following lipopolysaccharide stimulation, cytokine production is downregulated (4). Recently, it has been demonstrated that SP-A can also impact the adaptive immune system by altering $\mathrm{T}$-cell function (5). The ability of SP-A to promote or attenuate a proinflammatory response may be due to its ability to activate different cell surface signaling molecules and to differentially activate T-cells $(6,7)$. SP-A has gene transcription interactions that also result in the activation or inhibition of the nuclear transcription factor NF- $\mathrm{KB}(8,9)$. Finally, the evolution of SP-A argues that it likely has critical functions in humans. Specifically, genetic complexity increases from rodents with one SP-A gene, primates with 2 SP-A genes (SP-A1 and SP-A2), and humans with splice variants and sequence variability of SP-A1 and SP-A2 (10). Polymorphisms of the human SP-A genes are associated with pediatric diseases $(11,12)$.

Previously, our laboratory demonstrated that maternal SP-A is critical for newborn survival in a nonhygienic environment (corn dust bedding, i.e., heavy microbial and endotoxin burden (13)). Histological examination of SP-A null pups exposed to the nonhygienic environment demonstrated neutrophil infiltration in the stomach and small intestines, and peritoneal cultures demonstrated bacterial peritonitis (Bacillus sp. and Enterococcus sp.). These findings were not detected in wildtype (WT) pups in the nonhygienic environment or SP-A null pups reared a semisterile environment. A heterozygous breeding strategy showed that a SP-A heterozygote dam improved the survival of her SP-A null offspring in this environment. Thus, the protective effect of maternal SP-A on SP-A null offspring could have occurred in utero and or postnatally via ingestion of milk (13).

Mothers' milk contains immunoglobulins that have the potential for environmental antigen specificity via stimulation of mothers' intestinal or respiratory mucosal surfaces. For example, when respiratory syncytial virus (RSV) interacts with mothers' respiratory tract, milk secretory $\operatorname{IgA}$ (sIgA) specific for RSV polypeptides can be detected (14). Mucosal immunization with HIV peptides conjugated to keyhole limpet hemocyanin (KLH), results in stimulus-specific immunoglobulins in goat colostrum (15). The creation of milk sIgA specific for environmental antigens requires intact innate and adaptive immune systems and the bronchomammary or enteromammary circulations (16). Importantly, adult mice exposed to our laboratory's nonhygienic environment have increased lung antigen presenting cells in the absence of a cytokine inflammatory response (17). Respiratory SP-A, therefore, has the potential to affect milk sIgA. 
Several cytokines have been identified in human and murine milk, however, the impact of these proteins on the newborn intestine is just beginning to be elucidated (18). To date, most identified milk cytokines are antiinflammatory in nature, thus their function has been hypothesized to have immunomodulating effects, assisting in switching from $\mathrm{T}$ helper cell subset type 1 (TH1) to TH2 (19). The impact of common infectious exposures or changes in the immune system on milk cytokines is largely unknown.

We hypothesized that SP-A can promote environment-specific sIgA and an immunoprotective cytokine profile within mother's milk. SP-A null and WT murine dam-pup dyads and two environmental exposures were utilized in this study. An intranasal (i.n.) exposure to a novel T-cell-dependent antigen, 2,4-dinitrophenyl keyhole limpet hemocyanin (DNP-KLH), was used to evaluate if SP-A impacts milk sIgA concentration and antigen specificity. A subchronic exposure to a complex microbe and endotoxin rich nonhygienic environment (corn dust bedding) was also used to determine if SP-A impacts milk sIgA concentration and environment-specific sIgA production. Additionally, maternal local (lung lavage cells) and systemic responses (milk and serum cytokines) to the nonhygienic environment were assessed. The impact of maternal SP-A on newborn health via mothers' milk was assessed using a cross-fostering strategy during a nonhygienic environmental exposure. Our results strongly indicate that maternal SP-A can impact milk sIgA levels and cytokines, which may affect newborn health.

\section{RESULTS}

\section{DNP-KLH Exposure and Milk slgA}

Milk was obtained on postnatal day 2 (PND2) from i.n. diluent or DNP-KLH exposed dams for sIgA measurement. The milk sIgA levels from diluent exposed WT and SP-A null dams were $511 \pm 149 \mu \mathrm{g} / \mathrm{ml}$ and $491 \pm 110 \mu \mathrm{g} / \mathrm{ml}$, respectively. The milk sIgA levels from DNP-KLH exposed WT and SP-A null dams were $850 \pm 149 \mu \mathrm{g} / \mathrm{ml}$ and $864 \pm 111 \mu \mathrm{g} / \mathrm{ml}$, respectively. ANOVA analysis demonstrated no significant difference among all groups $(P=0.1844, n=4$ animals/ group).

Semiquantitative levels for DNP-KLH-specific milk sIgA from diluent and DNP-KLH exposed WT and SP-A null dams were determined using DNP-KLH-coated enzymelinked immunosorbent assay (ELISA) plates. ANOVA analysis demonstrated significant differences between these four groups $(P=0.0228)$. Further, a pairwise $t$-test demonstrated the average $\mathrm{OD}_{450} / \mu \mathrm{g}$ protein from DNP-KLH treated SP-A null dams was significantly lower $(P=0.027)$ when compared with the DNP-KLH-treated WT dams (Figure 1).

\section{Nonhygienic Environment Exposure and Milk slgA}

Milk sIgA from PND2 WT and SP-A null dams exposed to the control and the nonhygienic environments was quantified (Figure 2). ANOVA analysis demonstrated a significant difference between these four groups $(P<0.0001)$. Pairwise $t$-tests demonstrated the nonhygienic environment was associated with

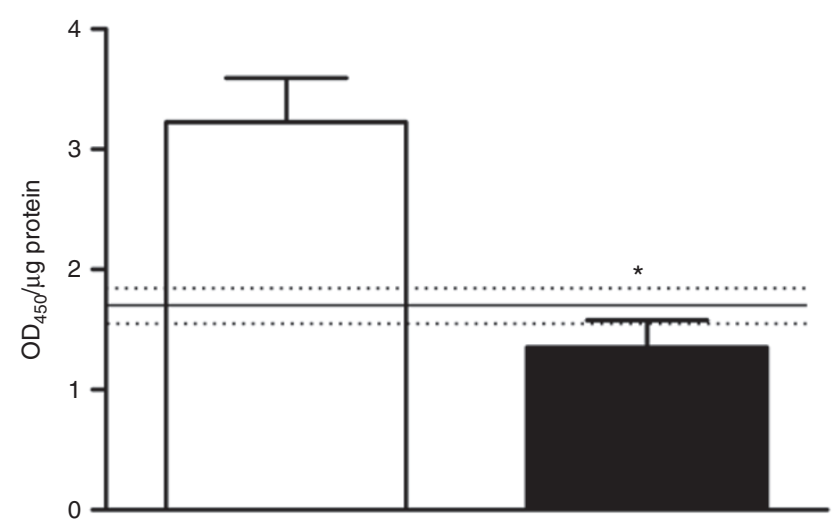

Figure 1. Semiquantitative measurements of milk slgA specific for 2,4-dintirophenyl keyhole limpet hemocyanin (DNP-KLH) from wild-type (WT) (white) and surfactant protein A (SP-A) null (black) dams. The mean and SEM of the $\mathrm{OD}_{450} / \mathrm{\mu g}$ protein of diluent-exposed WT and SP-A mice are demonstrated by solid and dashed lines, respectively. Following DNP-KLH exposure SP-A null milk slgA measured significantly less ( $\left.{ }^{*} P<0.05\right)$ compared to WT milk $\left(1.354 \pm 0.224 \mathrm{OD}_{450} / \mu \mathrm{g}\right.$ protein and $3.225 \pm 0.368 \mathrm{OD}_{450} / \mu \mathrm{g}$ protein, respectively). Milk samples were diluted 1:500. Data are the mean \pm $\mathrm{SEM}, n=6-8 \mathrm{mice} /$ group.

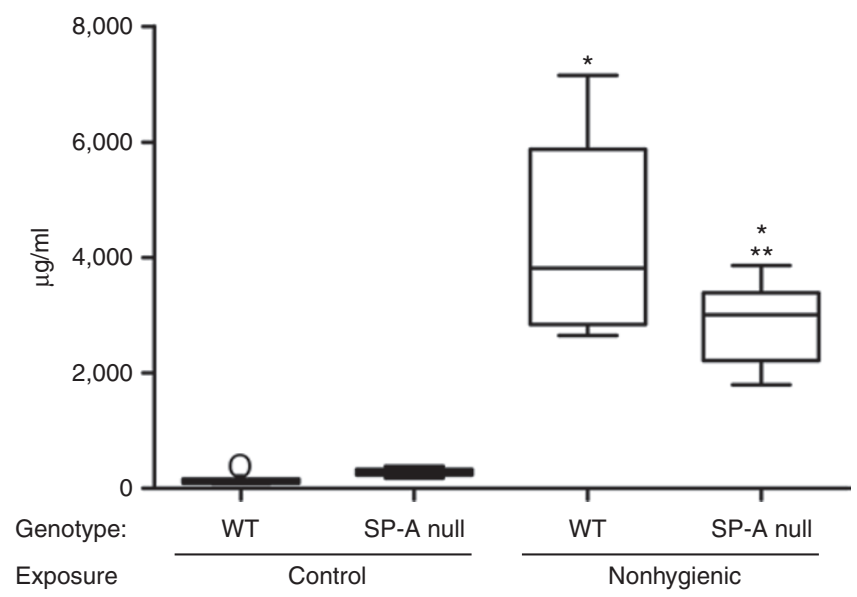

Figure 2. Milk slgA concentrations from control and nonhygienic environment-exposed wild-type (WT) and surfactant protein A (SP-A) null dams ( $n=7-8 /$ group). ANOVA demonstrated a significant $(P<0.0001)$ difference between these groups. WT and SP-A null dams exposed to the nonhygienic environment had significantly higher concentrations of milk $\operatorname{slgA}\left({ }^{*} P<0.0001\right)$ compared to the same genotype dams in the control environment. Further, the SP-A null milk contained significantly less slgA in the nonhygienic environment than the WT milk in the same environment ( $\left.{ }^{* *} P=0.0044\right)$. Lines within the box represent the group median, the box represents the upper and lower limits of the quartiles, the "whiskers" identify the most extreme data value not more than 1.5 times the interquartile range. Potential outliers are identified by a circle.

a significant increase in milk sIgA levels $(P<0.0001)$ when compared with control environment dams of the same strain. WT dams produced significantly more milk sIgA compared with SP-A null dams in the nonhygienic environment $(P=0.0044)$.

Semiquantitative levels of nonhygienic environment-specific milk sIgA were assessed using corn dust solution-coated ELISA plates and serial dilutions. Both strains of mice demonstrated 
Table 1. Signal intensity fold change ${ }^{a}$ in milk cytokines referenced to WT mice

\begin{tabular}{lccc}
\hline Cytokine & $\begin{array}{c}\text { SP-A null } \\
\text { control }^{b}\end{array}$ & WT nonhygienic & $\begin{array}{c}\text { SP-A null } \\
\text { nonhygienic }^{c}\end{array}$ \\
\hline IL-1 $\alpha$ & $>2.5$ & ND & $>2.5$ \\
IL-3 & ND & ND & $>1.5$ \\
IL-9 & ND & $>1.5$ & $>2.5$ \\
IL-12p70 & ND & ND & $>1.5$ \\
IL-13 & ND & ND & $>1.5$ \\
BLC & ND & ND & $>1.5$ \\
G-CSF & ND & $>3.5$ & $<0.5$ \\
I-TAC & ND & ND & $>1.5$ \\
KC & $>4.5$ & $>4.5$ & $<0.5$ \\
Leptin & ND & ND & $>1.5$ \\
MCP-1 & ND & $>2.5$ & ND \\
MCSF & ND & ND & $>2.5$ \\
SDF-1 & ND & ND & $>4.5$ \\
TCA-3 & ND & ND & $>1.5$ \\
TECK & ND & ND & $>1.5$ \\
STNF-RII & ND & $>1.5$ & ND
\end{tabular}

BLC, B-lymphocyte chemoattractant; G-CSF, granulocyte colony-stimulating factor; IL, interleukin; I-TAC, interferon-inducible T-cell a chemoattractant; MCP-1, monocyte chemoattractant protein-1; MCSF, macrophage-stimulating factor; SDF-1, stromalderived factor-1; STNF-RII, soluble TNF receptor II; TCA-3, T-cell activation protein-3; TECK, thymus-expressed chemokine; WT, wild type.

asignal intensity fold change is categorized as $\langle 0.5,>1.5,>2.5,>3.5,>4.5$-fold difference or not different (ND, fold difference within 0.49 and 1.49) when compared with WT mice. The signal intensity of each cytokine spot was performed in duplicate, the background was subtracted, and data averaged (signal intensity data collection performed by RayBiotech). $n=5$ pooled samples/group. 'Referenced to WT mice exposed to the control environment. 'Referenced to WT mice exposed to the nonhygienic environment.

the presence of milk sIgA specific to nonhygienic environmental antigens at titers $\leq 1: 200$ compared with control environment-exposed mice of both strains $(P>0.05, n=4$ mice/ group, data not shown). There were no significant differences in the semiquantitative sIgA levels between WT and SP-A null nonhygienic environment-exposed mice at titers ranging from $1: 1,000$ to $1: 10$.

\section{Nonhygienic Environment Exposure and Milk Cytokines}

Milk from PND2 dams exposed to the control or the nonhygienic environment was assessed using a protein array. Those cytokines that met the physiologically meaningful criteria are presented (Table 1). Two proteins, IL- $1 \alpha$ and KC (GRO$\alpha /$ CXCL1), were increased in the absence of SP-A in the control environment. This comparison was performed twice with consistent results. Five milk proteins were increased when WT mice were exposed to the nonhygienic environment compared with the control environment. Finally, the collective impact of the nonhygienic environment, and the absence of SP-A was examined. Twelve proteins met criteria for physiologically meaningful increases in the SP-A null milk, and two cytokines were decreased in the SP-A null milk, compared with WT milk.
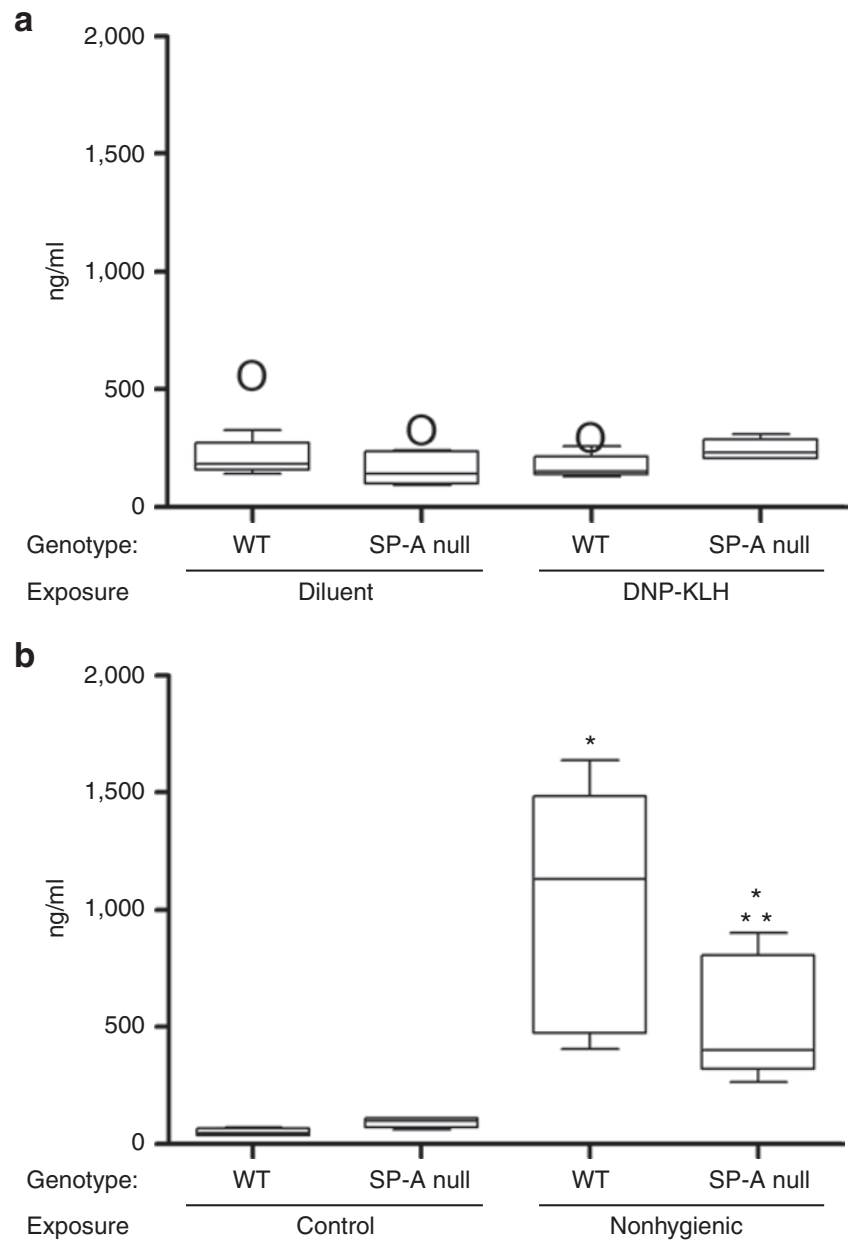

Figure 3. Impact of 2,4-dintirophenyl keyhole limpet hemocyanin (DNP$\mathrm{KLH}$ ) and the nonhygienic environment on lung lavage IgA concentration. (a) Lung IgA concentrations from wild-type (WT) and surfactant protein A (SP-A) null dams exposed to diluent and DNP-KLH ( $n=6-8 /$ group). ANOVA demonstrated no significant difference due to either dam genotype or exposure. (b) Lung IgA concentrations from control and nonhygienic environment-exposed WT and SP-A null dams ( $n=5-7 /$ group). ANOVA demonstrated significant $(P<0.0001)$ differences between these groups. WT and SP-A null dams exposed to the nonhygienic environment produced significantly more $\lg A\left({ }^{*} P<0.02\right)$ compared with the same genotype dams in the control environment. Further, the SP-A null dams produced significantly less IgA in the nonhygienic environment than the WT dams in the same environment $(* * P=0.0093)$. Lines within the box represent the group median, the box represents the upper and lower limits of the quartiles, the "whiskers" identify the most extreme data value not more than 1.5 times the interquartile range. Potential outliers are identified by a circle.

\section{Nonhygienic Environmental Exposure and Serum Cytokines}

Serum from PND7 WT and SP-A null dams exposed to either the control or nonhygienic environments ( $n=5$ mice/group) were assessed using a protein array. There were no differences between genotypes or exposures in any serum cytokine which previously demonstrated a meaningful change in milk levels, with the exception of serum leptin and granulocyte colonystimulating factor (G-CSF). Both strains of control environment exposed dams demonstrated similar semiquantitative levels of serum leptin, and both strains had a similar physiologically meaningful increase in leptin during the nonhygienic exposure. SP-A null serum G-CSF was decreased $>0.5$-fold 
compared with WT serum under control conditions. In the nonhygienic environment, WT serum G-CSF was increased 2.66-fold compared with control WT serum. Conversely, SP-A null serum G-CSF levels remained unchanged in the nonhygienic environment compared with the control environment.

Lungs Response to DNP-KLH and the Nonhygienic Environment Lung lavage total cell number and cell type from PND7 dams following the DNP-KLH, diluent, nonhygienic environment, or control environment exposures did not differ significantly ( $n=4-7$ animals/group, ANOVA analysis, data not shown).

Lung lavage fluid IgA levels from PND7 WT and SP-A null dams exposed to diluent or DNP-KLH did not differ significantly ( $P=0.5945$, ANOVA) (Figure 3a). Nonhygienic environment-exposed dams demonstrated a significant increase in lung IgA compared with control dams $(P<0.0001$, ANOVA) (Figure $3 \mathrm{~b}$ ). Pairwise $t$-tests demonstrated the nonhygienic environment was associated with a significant increase in lung
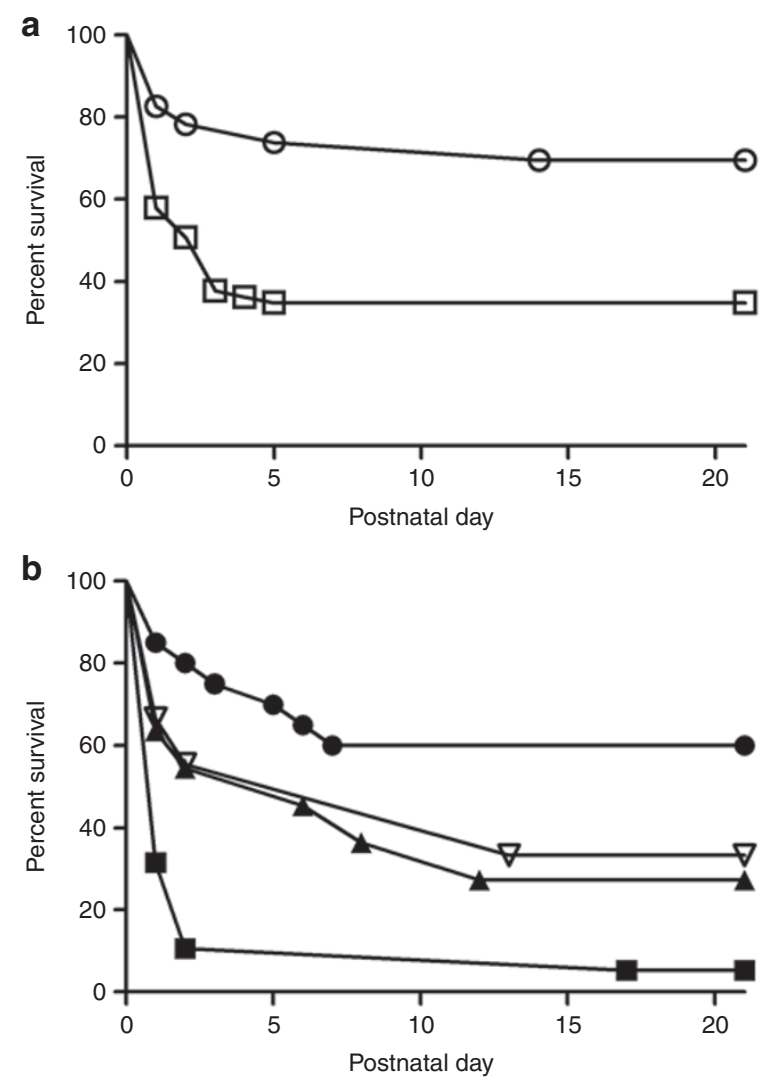

Figure 4. Kaplan-Meier survival curves of wild-type (WT) and surfactant protein A (SP-A) null pup survival in the nonhygienic environment. (a) Biological dam-pup pairs. SP-A null pup survival (open square, $n=$ 69) until weaning (PND21) is significantly reduced compared to WT pup survival (open circle, $n=23)(\mathrm{HR}=2.08,95 \% \mathrm{Cl}(1.19,3.67), P=0.01)$. (b) The survival of cross-fostered pups or biological pups of a fostering dam in the nonhygienic environment is presented. The survival of cross-fostered SP-A null pups reared by a WT foster dam (solid triangle, $n=11$ ) tended to be greater when compared with their biological littermates reared by their biological SP-A null fostering dam (solid square, $n=19$ ). Survival of WT pups fostered to a SP-A null dam (open triangle, $n=9$ ) was similar to the opposite fostering situation (solid triangle). WT pups reared by their biological WT fostering dam had the best survival (solid circle, $n=20$ ).
IgA levels in both the WT $(P<0.0001)$ and SP-A null dams $(P$ $=0.0128)$ compared with control environment-exposed dams. Further, WT dams produced significantly more lung IgA compared with SP-A null dams under the nonhygienic conditions $(P=0.0093)$.

\section{Newborn Survival in the Nonhygienic Environment}

Litter size at birth during the biological dam-biological pup survival assessment averaged $6.6 \pm 1.3$ and $6.8 \pm 2.1$ pups/litter (WT and SP-A null litters, respectively). Litter size at birth during the cross-fostering experiment averaged $6.6 \pm 0.69$ and $6.9 \pm 0.67 \mathrm{pups} / \mathrm{litter}$ (WT and SP-A null litters, respectively). Of the breeders paired for the cross-fostering experiment, $50 \%$ had litters that met the size and timing criteria outlined in the methods section.

Pup survival Kaplan-Meier curves with biological dampup dyads are presented (Figure 4a). Pups that experienced a cross-fostering situation are presented separately (Figure $4 \mathrm{~b}$ ). Using proportional hazards regression with a Gaussian frailty term for birth litter, SP-A null pups had a statistically significant increase $(P=0.01)$ in hazard for death compared with WT pups when reared by their biological dams (Figure 4a). Survival for the biological offspring of a WT fostering dam was $69 \%$ compared with $80 \%$ in the absence of cross-fostered pups. Survival for SP-A null pups decreased from 34.7 to $5 \%$ when fostered pups were added to a litter. Due to the impact of crossfostering on pup survival, this portion of data analysis included only those dam-pup dyads that participated in a cross-fostered situation. Since a small number of pups were available for this portion of the study, only a descriptive evaluation of the crossfoster survival curves can be made. The impact of WT milk on SP-A null pups in the nonhygienic environment was an increase in survival from $5.2 \%$ ( 1 in 19 SP-A null pups, biological offspring of a fostering dam) to $27.3 \%$ (3 in 11 SP-A null pups cross-fostered to a WT dam) (Figure 4b). For comparison, $33.3 \%$ of WT pups cross-fostered to a SP-A null dam survived ( 3 in 9 pups).

\section{DISCUSSION}

Given the known functions of SP-A in the respiratory track and an adaptive immune system that allows for milk sIgA specific for respiratory antigens, we hypothesized that SP-A may be critical for optimal immunoprotective properties within mothers' milk. Our data demonstrate different milk immunoglobulin responses depending on the respiratory exposure. The first experiment utilized a novel T-cell-dependent antigen, KLH. KLH has previously been used to assess milk immunoglobulin production in rodent models, yet the impact of SP-A with this stimulus has not been studied (20). The nonhygienic environment has been characterized as a complex microbial and endotoxin-rich inhalational exposure, components to which SP-A can bind directly to and enhance phagocytosis $(5,13)$. Total milk and lung IgA content is not affected by the SP-A null genotype or exposure to DNP-KLH, yet maternal SP-A allows for optimal DNP-KLH-specific milk sIgA production. Maternal SP-A enhances but is not required for the production 
of milk and lung IgA when exposed to the nonhygienic environment. Further, SP-A is not necessary for milk sIgA specific for antigens present in the nonhygienic environment.

While commonly considered a protein with innate immune system functions, SP-A also affects the adaptive immune system. Recent studies demonstrate increased T-cell activation and proliferation in the presence of SP-A following low dose lipopolysaccharide or ovalbumin stimulation (7). Conversely, these functions are attenuated when higher stimulus doses are used. Applying our present studies to this literature, the T-celldependent DNP-KLH exposure may be functioning as a weak immune stimulus in the presence of SP-A. This mechanism could account for the production of antigen-specific milk sIgA in WT but not SP-A null mice. The more complex nonhygienic environment, by virtue of its ability to activate the immune system by several mechanisms (i.e., direct microbe-antigen presenting cell interactions), may have allowed the production of milk sIgA specific to the stimulus independent of SP-A.

To date, there is little in the literature with respect to the impact of SP-A on immunoglobulin production. Murine studies suggest SP-A has a role in reducing ovalbumin-induced cytokines and IgE levels (3). Our results are in contrast to this allergen model as SP-A is associated with enhanced lung and milk IgA production. In both laboratory conditions, however, SP-A appears to contribute to the host's immune system health by virtue of its ability to promote or attenuate an immune response.

The ability of SP-A to modulate a cytokine profile appears to depend on the initial stimulus, the type of cell(s) and cell surface receptors stimulated, and the state of cell activation (21). It has been postulated that the interaction of SP-A with cell signaling proteins, such as SIRP $\alpha$ inhibits activation of the transcription factor NF- $\mathrm{KB}$, thus preventing the production of proinflammatory mediators (6). Conversely, interaction of SP-A with the cell-signaling proteins calreticulin/CD91 results in activation of NF- $\mathrm{KB}$ leading to an inflammatory response. The current study demonstrates a baseline proinflammatory milk cytokine profile in the absence of SP-A. Further, both the nonhygienic environment and SP-A null genotype independently, and synergistically result in milk with a proinflammatory cytokine profile. This inflammatory response is similar to what is described in microbe-induced SP-A null lung injury murine models $(2,22)$.

There is limited data with respect to the source of milk cytokines and their physiologic effect on newborns. G-CSF is present in human milk and the G-CSF receptor is present in newborn intestines $(23,24)$. Further, cytokines and chemokines may be functional postingestion (25). Thus, milk proteins have the potential for physiologic function in the newborn. Milk cytokines are increased in the cell-free portion of human milk following RSV infection, and milk leukocyte cytokine production is induced by lipopolysaccharide $(26,27)$. The prominent proinflammatory cytokine profile in SP-A null milk and simultaneous lack of proinflammatory cytokines in serum is consistent with human studies during an RSV infection (27). Thus, our data further support literature, which suggest systemic maternal stimuli can preferentially alter milk cytokine levels compared with serum levels.

The cross-fostering strategy attempted to evaluate the impact of WT milk on SP-A null pup survival. This method, however, was met with many challenges limiting the number of litters available for study. The trends identified, however, suggest milk from WT mice may improve SP-A null pup survival. Future utilization of less extreme conditions or end points may help clarify the significance of maternal SP-A and milk immunoprotective properties on newborn health.

Milk passive immunity specific for environmental antigens has been referred to as a "natural vaccination." (28) Pediatric diseases, including respiratory distress syndrome of the newborn and otitis media, are associated with human SP-A polymorphisms $(11,12)$. The impact of maternal SP-A on newborn health via milk immunoprotective properties has to date not been explored. We hypothesized that maternal SP-A would have an immunoprotective effect on milk sIgA function and cytokine profile. Given prior experience with SP-A null dampup dyads in the nonhygienic environment, this murine model was again utilized to address this hypothesis. These studies demonstrate maternal SP-A attenuates a proinflammatory cytokine profile in milk yet has a mixed effect on milk immunoglobulin levels and specificity. Specifically, maternal SP-A is not required for environment-specific passive immunity when the stimulus is complex. The passive immunity present in SP-A null milk is not protective to SP-A null newborns in the nonhygienic environment. Conversely, DNP-KLH antigen-specific milk IgA production is optimized by maternal SP-A. The SP-A null genotype is associated with milk containing a proinflammatory cytokine profile and increased newborn mortality during a microbe rich exposure. While the physiologic effect of milk cytokines ingested by a newborn is largely unknown, these data suggest proinflammatory milk cytokines due to a lack of maternal SP-A may adversely affect newborn health.

\section{METHODS}

\section{Animals}

Breeding pairs of $\mathrm{C} 3 \mathrm{HeB} / \mathrm{FeJ}$ (Jackson Laboratories, Bar Harbor, ME) and SP-A null mice on the same background (original mutated mice provided by Dr. F. McCormack, University of Cincinnati) at $7-9 \mathrm{wk}$ of age were established. For each experiment, age-matched breeding pairs were analyzed. Mice were provided food and water ad libitum. All procedures were performed according to the protocols approved by the University of Minnesota Animal Care and Use Committee.

\section{DNP-KLH Immunization of Mice}

Eight-week-old female mice were exposed to $30 \mu \mathrm{l}$ i.n. DNP-KLH $(1 \mathrm{mg} / \mathrm{ml}$ in water) or $30 \mu \mathrm{l}$ i.n. deionized water (diluent) on days 1 and 14 after pairing. Isoflurane via a nose cone provided brief anesthesia.

\section{Nonhygienic Environmental Exposure}

This exposure has been described in detail previously (13). Briefly, corn dust was collected and combined 1:1 with our institutions regular semisterile cage bedding (7092, Harlan Laboratories, Madison, WI) while the control environment consisted of the semisterile cage bedding. Adult mice were acclimated to the corn dust bedding for $2 \mathrm{wk}$ prior to pairing. The mice used for milk collection were exposed to this environment for $\geq 12 \mathrm{wk}$. When milk collection was required, breeder pairs were placed into control bedding $24-48 \mathrm{~h}$ prior to the delivery of a litter to allow successful delivery, nursing, and milk collection without the 
stress of the nonhygienic environment. These mice were then returned to the nonhygienic environment for lung lavage collection.

\section{Dam Sample Collection}

Milk was collected on PND2 by removing the dam from her litter for $1 \mathrm{~h}$. Fifteen minutes prior to milking, intraperitoneal oxytocin (2U/100 $\mu \mathrm{l}$ phosphate-buffered saline) was administered. Under isoflurane anesthesia, an adapted breast pump was used to induce milk let-down followed by manual massage resulting in 50-200 $\mu \mathrm{l} \mathrm{milk/}$ animal. Lung lavage fluid and alveolar cells were collected on PND7 and processed as previously described with a lavage volume of $3 \mathrm{ml}$ (17). Serum was collected on PND7 by allowing freshly pooled blood to sit for $30 \mathrm{~min}$ at RT, separated by centrifugation at $2,500 \mathrm{rpm}$ at $4{ }^{\circ} \mathrm{C}$, and then stored at $-80^{\circ} \mathrm{C}$ until used.

\section{Milk Processing}

Phenylmethylsulfonylfluoride $(10 \mu \mathrm{l}$ of $100 \mathrm{mmol} / \mathrm{l})$ was added to each fresh milk sample to inhibit endogenous proteolytic activity (29). The milk was diluted with deionized water (1:1), centrifuged at $10,000 \mathrm{rpm}$ for $10 \mathrm{~min}$ at $4{ }^{\circ} \mathrm{C}$. The clarified portion and the pellet were stored at $-80^{\circ} \mathrm{C}$.

\section{Total and Antigen-Specific Immunoglobulin Determination}

A mouse IgA ELISA quantitation set (Bethyl Laboratories, Montgomery, TX) was used according to the manufacturer's protocol. Serial dilutions of milk and lung lavage fluid were tested to identify the optimal dilution within the linear portion of the standard curve. Samples and standards were assayed in duplicate. The horseradish peroxidase enzyme conjugate antibody was diluted 1:25,000-75,000, optimized for each study. Results were expressed as total IgA ng/ml with a low level of detection equal to $15.625 \mathrm{ng} / \mathrm{ml}$.

To detect DNP-KLH-specific IgA, ELISA plates were coated overnight with DNP-KLH $(10 \mu \mathrm{g} / \mathrm{ml})$ diluted in carbonate coating buffer at $4{ }^{\circ} \mathrm{C}$ prior to washing and blocking. To detect IgA specific for nonhygienic environmental antigens, a corn dust extract was prepared as previously described (30). Briefly, $0.5 \mathrm{~g}$ dust was incubated with $5 \mathrm{ml}$ Hanks' balanced salt solution at $10^{\circ} \mathrm{C}$ for $1 \mathrm{~h}$. Large particulates were separated by straining through glass wool. One milliliter Trisbuffered saline with $0.05 \%$ azide was added, and the solution was sonicated to ensure the solution was sterile. The ELISA plates were coated with corn dust extract $(10 \mu \mathrm{g} / \mathrm{ml})$ diluted in carbonate coating buffer at $4{ }^{\circ} \mathrm{C}$ overnight prior to washing and blocking. Total protein levels were quantified using a CB Protein assay kit (Calbiochem, EMD Biosciences, La Jolla, CA). The results were expressed as $\mathrm{OD}_{450}$ or the lowest detectible titer above unexposed control animals.

\section{Milk and Serum Cytokine Determination}

A protein array method was utilized for pooled milk and pooled serum samples from WT and SP-A null control or nonhygienic environment-exposed mice. The mouse inflammation antibody array G1 series (RayBiotech, Norcross, GA) was used according to the manufacturer's protocol. This technology allows simultaneous detection of 40 cytokines using an ELISA-based method resulting in relative concentrations based on signal intensity. $A \geq 1$. 5-fold increase or $\geq 0.5$ fold decrease (calculated as a $\geq 50 \%$ decrease) in signal intensity comparing one cytokine between two groups is considered physiologically meaningful per the manufacture. Further, only those proteins with values greater than the internal negative control and less than the internal positive control are presented. Human milk protein quantification, using ELISA techniques, demonstrates moderate to strong correlation with this type of array (31).

\section{Cross-Fostering}

Breeder mice were paired such that WT and SP-A null litters were born within $8 \mathrm{~h}$ of each other. Once it was determined the pups were nursing well at $<24 \mathrm{~h}$ of life, two to three pups were cross-fostered so that the original litter size was approximated. The pups were marked for identification. Each cross-foster dam was allowed to keep at least 3 of her biological offspring.

\section{Statistical Analysis}

The data are summarized as mean \pm SEM. Pairwise differences of group means were performed using a $t$-test. ANOVA followed by pairwise $t$-tests were used to compare more than two groups using PRISM5, GraphPadSoftware (La Jolla, CA) or SAS/STAT software, Version 9.333 for Linux, SAS Institute (Cary, NC). Time-to-event data are presented as Kaplan-Meier survival curves and analyzed using proportional hazards regression with a Gaussian frailty term for birth litter to account for within-litter correlation in survival times (SAS/ STAT software). Significance was considered for $P$ values $<0.05$.

\section{STATEMENT OF FINANCIAL SUPPORT}

This study was funded in part by a March of Dimes Research Grant, White Plains, NY and by the National Center for Advancing Translational Sciences of the National Institutes of Health, Bethesda, MD, award number 5UL1TR000114-03.

Disclosure: The authors have no financial disclosures to make or conflicts of interest.

\section{REFERENCES}

1. Wright JR. Immunomodulatory functions of surfactant. Physiol Rev 1997;77:931-62.

2. LeVine AM, Whitsett JA, Gwozdz JA, et al. Distinct effects of surfactant protein A or D deficiency during bacterial infection on the lung. J Immunol 2000;165:3934-40.

3. Pastva AM, Mukherjee S, Giamberardino C, et al. Lung effector memory and activated $\mathrm{CD} 4+\mathrm{T}$ cells display enhanced proliferation in surfactant protein A-deficient mice during allergen-mediated inflammation. J Immunol 2011;186:2842-9.

4. McIntosh JC, Mervin-Blake S, Conner E, Wright JR. Surfactant protein A protects growing cells and reduces TNF-alpha activity from LPS-stimulated macrophages. Am J Physiol 1996;271(2 Pt 1):L310-9.

5. Nayak A, Dodagatta-Marri E, Tsolaki AG, Kishore U. An Insight into the Diverse Roles of Surfactant Proteins, SP-A and SP-D in Innate and Adaptive Immunity. Front Immunol 2012;3:131.

6. Gardai SJ, Xiao YQ, Dickinson M, et al. By binding SIRPalpha or calreticulin/CD91, lung collectins act as dual function surveillance molecules to suppress or enhance inflammation. Cell 2003;115:13-23.

7. Mukherjee S, Giamberardino C, Thomas J, et al. Surfactant protein A integrates activation signal strength to differentially modulate $\mathrm{T}$ cell proliferation. J Immunol 2012;188:957-67.

8. Alcorn JF, Wright JR. Surfactant protein A inhibits alveolar macrophage cytokine production by CD14-independent pathway. Am J Physiol Lung Cell Mol Physiol 2004;286:L129-36.

9. Wu Y, Adam S, Hamann L, et al. Accumulation of inhibitory kappaB-alpha as a mechanism contributing to the anti-inflammatory effects of surfactant protein-A. Am J Respir Cell Mol Biol 2004;31:587-94.

10. Floros J, Wang G, Mikerov AN. Genetic complexity of the human innate host defense molecules, surfactant protein A1 (SP-A1) and SP-A2-impact on function. Crit Rev Eukaryot Gene Expr 2009;19:125-37.

11. Jo HS, Cho SI, Chang YH, Kim BI, Choi JH. Surfactant protein A associated with respiratory distress syndrome in Korean preterm infants: evidence of ethnic difference. Neonatology 2013;103:44-7.

12. Pettigrew MM, Gent JF, Zhu Y, et al. Association of surfactant protein A polymorphisms with otitis media in infants at risk for asthma. BMC Med Genet 2006;7:68.

13. George CL, Goss KL, Meyerholz DK, Lamb FS, Snyder JM. Surfactantassociated protein A provides critical immunoprotection in neonatal mice. Infect Immun 2008;76:380-90.

14. Tsutsumi H, Honjo T, Nagai K, Chiba Y, Chiba S, Tsugawa S. Immunoglobulin A antibody response to respiratory syncytial virus structural proteins in colostrum and milk. J Clin Microbiol 1989;27:1949-51.

15. Dorosko SM, Ayres SL, Connor RI. Induction of HIV-1 MPR(649684)-specific IgA and IgG antibodies in caprine colostrum using a peptidebased vaccine. Vaccine 2008;26:5416-22. 


\section{SP-A influence on milk

16. Newburg DS, Walker WA. Protection of the neonate by the innate immune system of developing gut and of human milk. Pediatr Res 2007;61:2-8.

17. George CL, White ML, Kulhankova K, et al. Early exposure to a nonhygienic environment alters pulmonary immunity and allergic responses. Am J Physiol Lung Cell Mol Physiol 2006;291:L512-22.

18. Garofalo R. Cytokines in human milk. J Pediatr 2010;156:Suppl 2:S36-40.

19. Lönnerdal B. Nutritional and physiologic significance of human milk proteins. Am J Clin Nutr 2003;77:1537S-43S.

20. Becker MI, De Ioannes AE, León C, Ebensperger LA. Females of the communally breeding rodent, Octodon degus, transfer antibodies to their offspring during pregnancy and lactation. J Reprod Immunol 2007;74:68-77.

21. Wright JR. Immunoregulatory functions of surfactant proteins. Nat Rev Immunol 2005;5:58-68.

22. Harrod KS, Trapnell BC, Otake K, Korfhagen TR, Whitsett JA. SP-A enhances viral clearance and inhibits inflammation after pulmonary adenoviral infection. Am J Physiol 1999;277(3 Pt 1):L580-8.

23. Calhoun DA, Lunoe M, Du Y, Christensen RD. Granulocyte colony-stimulating factor is present in human milk and its receptor is present in human fetal intestine. Pediatrics 2000;105:e7.
24. Maheshwari A, Christensen RD, Calhoun DA. ELR+ CXC chemokines in human milk. Cytokine 2003;24:91-102.

25. Calhoun DA, Lunøe M, Du Y, Staba SL, Christensen RD. Concentrations of granulocyte colony-stimulating factor in human milk after in vitro simulations of digestion. Pediatr Res 1999;46:767-71.

26. Skansén-Saphir U, Lindfors A, Andersson U. Cytokine production in mononuclear cells of human milk studied at the single-cell level. Pediatr Res 1993;34:213-6.

27. Sone S, Tsutsumi H, Takeuchi R, et al. Enhanced cytokine production by milk macrophages following infection with respiratory syncytial virus. J Leukoc Biol 1997;61:630-6.

28. Zinkernagel RM. On natural and artificial vaccinations. Annu Rev Immunol 2003;21:515-46.

29. Parr EL, Bozzola JJ, Parr MB. Purification and measurement of secretory IgA in mouse milk. J Immunol Methods 1995;180:147-57.

30. Wohlford-Lenane CL, Deetz DC, Schwartz DA. Cytokine gene expression after inhalation of corn dust. Am J Physiol 1999;276(5 Pt 1):L736-43.

31. Kverka M, Burianova J, Lodinova-Zadnikova R, et al. Cytokine profiling in human colostrum and milk by protein array. Clin Chem 2007;53:955-62. 